\title{
Human Photosynthesis: A Turning Point in the Understanding and Treatment of Alzheimer's Disease
}

\author{
Arturo Solís Herrera ${ }^{1}$, Jerzy Leszek ${ }^{2 *}$, María del Carmen Arias Esparza ${ }^{1}$, Ruth I Solís-Arias ${ }^{1}$, Paola E Solís-Arias ${ }^{1}$ and Martha P Solís-Arias ${ }^{1}$ \\ ${ }^{1}$ Human Photosynthesis Study Center, Arturo Solís Herrera, Poland \\ ${ }^{2}$ Medical University in Wroclaw, Poland
}

\begin{abstract}
More than 100 years have passed by since the first description of AD by Alois Alzheimer; however the patients, presently; are dying in the same way in spite the technological advance. Our finding of the previously unknown capacity of the melanin's human body to split and reform the water molecule, appointed as human photosynthesis by its analogies with photosynthesis in plants; constitutes a disruptive advance in the knowledge because means that glucose is not a source of energy, instead it is a biomass source only. If really glucose were a source of energy, then diabetic patients should be able to fly. It is out of discussion that glucose is the ideal building block; our body even makes nucleic acids with it. But energy, (anything that makes a change) is astonishingly taken from water. $A D$ is an energy problem, not a biomass trouble. In this speech we are presenting the concept, the basis and the amazing therapeutic results in Alzheimer's disease that the medical enhancement of Human Photosynthesis can give. Therefore the role of the CSF must be rethought due that water is so far the main source of energy of the CNS and the cerebral sulcus the best way to keep a close contact between neurons and water, therefore to the neuron cell water constitutes a source of energy that is easily affordable through the subarachnoid space and the ventricles. From now in ahead, the sacred role of glucose as source of energy is broken into small pieces. Human photosynthesis is a turning point in the understanding and treatment of the plague of the XXI century, the Alzheimer's Disease.
\end{abstract}

Keywords: Alzheimer's Disease; Human photosynthesis; Metabolical processes

\section{Introduction}

Photosynthesis is basically a black box, the internal mechanism of which is poorly understood. Photo-transduction or the transformation of the photonic energy (of light) into free chemical energy is a very difficult process to study, because, like other metabolical processes, photosynthesis proved highly sensitive to intervention [1]. Whenever the box's covering was pried off, the wheels inside stopped turning [2]. Outside the living cells all the molecules that take part in the photosynthetic processes must be rendered reactive, because were known to be highly inert.

In plants, photosynthesis is modulated by $\mathrm{CO}_{2}$ concentrations, light intensity, temperature; moisture, pressure, etc. At low light intensities, the rate of photosynthesis increased in proportion to the light, while this effect became less prominent at higher light intensities. After a certain point, the rate of photosynthesis reached a plateau and additional increases in light intensity were unable to promote the process any further.

Interestingly, comparing the effects of intermittent illumination, Otto Warburg found that high light intensities a certain amount of energy was able to decompose more carbonic acid (formula $\mathrm{H}_{2} \mathrm{CO}_{3}$-equivalently $\left.\mathrm{OC}(\mathrm{OH})_{2}{ }^{-}\right)$at intermittent illumination than at continuous illumination. The increase in efficiency depended on the rate of alternation between light and dark periods: at a rate of 8000 alternations per minute efficiency increase by almost $100 \%$, while at a rate of four alternations per minute an increase of only $10 \%$ was achieved. Perhaps due to after a dark period an increased concentration of decomposable substance would be available.

Photosynthetic assimilation could be reversibly inhibited by phenyl-urethane (Figure 1). Taking into account that the effect of anesthetics is due to changes in the membranes, the conclusion is that the slightest changes in these layers thus inhibits the process of photosynthetic assimilation, in plants and humans. Recall that aged patients sometimes awoke from a general anesthesia with Alzheimer's Disease.

The inhibitory effect of an anesthetic substance was stronger, the higher its adsorptive capacity (tendency to adhere to surfaces). Thereby photosynthesis reactions are surface dependent, in plants and humans. It is possible that light absorption itself is not sensitive to anesthetics; instead, the damage must involve some kind of surface-sensitive reaction.

\section{The Role of Neurotransmitters}

Expression of the appropriate neurotransmitters is essential for the

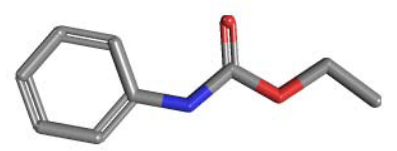

Figure 1: Phenyl-urethane molecule.

*Corresponding author: Arturo Solís Herrera, Human Photosynthesis Study Center, Av. Poland, Tel: +524499160048; E-mail: comagua2000@yahoo.com

Received May 04, 2013; Accepted June 17, 2013; Published June 20, 2013

Citation: Herrera AS, Leszek J, del Carmen Arias Esparza M, Solís-Arias RI, Solís-Arias PE, et al. (2013) Human Photosynthesis: A Turning Point in the Understanding and Treatment of Alzheimer's Disease. J Bioanal Biomed 5: 057060. doi:10.4172/1948-593X.1000079

Copyright: (c) 2013 Herrera AS, et al. This is an open-access article distributed under the terms of the Creative Commons Attribution License, which permits unrestricted use, distribution, and reproduction in any medium, provided the original author and source are credited. 
function of neural circuits [1]; however sufficient free chemical energy is fundamental at most. Neurons are able to change their transmitter phenotype as result and to deal with in the best way with variations mainly of the light levels in the environment. Variables other as humidity, temperature, $\mathrm{pH}$, food availability, age, sex, stress degree and so forth are important too, but the amount of light, visible and invisible; its important especially due to the relatively recent finding of the intrinsic property of melanin to split and re-form the water molecule, thereby melanin possess the unexpected capacity to transform the photonic energy into free chemical energy that is susceptible to be used by the eukaryotic cell.

Thereby it is not by chance that exposure of animal labs to different photoperiods, mimicking summer and winter day-lengths, is followed by meaningful changes at practically every organizational level, from cells to organs and systems.

It is possible to find significant variation even in the intracellular organelles owing to differences in the quantity of light, visible and invisible. Let's see some examples:

- The diffusion of oxygen dissolved in the aqueous compartment of the cell appears to be quite sensitive to decreasing temperature (and light), primarily because of a pronounced reduction in $\mathrm{DO}_{2}$ (diffusion coefficient) mediated by an elevation in cytoplasmic viscosity [2-4].

- The cytoplasmic viscosity increase during reductions in cell temperature (and light). In Nature usually a decrease of amount of light is accompanied with a reduction in temperature.

- Studies of eurythermal fishes showed that cold acclimation causes initial decreases in the rates of aerobic metabolism.

- Thermal acclimation of fishes was capable of inducing very dramatic changes in sub-cellular anatomy.

- During acclimation of crucian carp from $28^{\circ} \mathrm{C}$ to $2^{\circ} \mathrm{C}$, the mitochondrial density of red muscle fibers increased by $80 \%$. A documented fact for numerous other fish species.

- The cold-induced mitochondrial proliferation in red fibers muscle, from $25^{\circ} \mathrm{C}$ and $5^{\circ} \mathrm{C}$, mitochondria displaced $28.6 \%$ and $44.8 \%$ cell volume, respectively.

- In contemporary species related with species originated in ancient times, when the Earth temperature was higher than the current conditions, the mitochondria number is meaningful less than in species recently originated, for instance, in birds, that are dinosaur-related specie.

- Neurotransmitter expression switched in hypothalamic neurons it's not an isolated case. Transmitter switching occurred at several levels: at transcriptional level and was accompanied by changes in postsynaptic receptors. The very first common requirement at levels all is sufficient free chemical energy available, otherwise, the highly complex neuronal cell organization cannot carry out a proper neurotransmitter expression switching, owing to every single part of the large number of processes involved have a very first requirement in common during the entire process: available free chemical energy.

- During long days, there is a higher concentration of estradiol in the cerebrospinal fluid (CSF) than during short days [5].
The previously unknown capacity of human body to uses water as source of electrons is an astonishing finding that is a conceptual revolution of biblical proportions. The sacred role of glucose as source of energy now is broken into small pieces. Therefore human body build up its biomass arising from glucose, thereby carbohydrates (or meals) are just a source of carbon chains of different lengths, branching; combinations with other elements and so forth. We could say that glucose $\left(\mathrm{C}_{6} \mathrm{H}_{12} \mathrm{O}_{6}\right)$ is the perfect building block. Our body is able to synthesize even nucleic acids with it. But our body fulfills its energy needs by means of the unexpected and astonishing capacity of melanin to split and re-form the water molecule.

\section{Energy Definition}

It is not known what energy is. Is it a line? Is it a circle? Is it a wave? Is it a comma? Who knows? Energy is everything that is able to produce some change, whichever could be.

\section{Alzheimer's Disease}

It is clinically characterized by a deterioration of memory and other cognitive domains that leads to death within 3 to 9 years after diagnosis, being the most common form of dementia [5]

Although a battery of neuropsychological tests are often used in making a clinical diagnosis of Alzheimer's Diseases (AD), diagnosis still relies in clinical assessment [6]. This progressive neurodegenerative disorder estimated to affect 5.1 million individuals in 2007 in USA. Unfortunately there are no affordable biomarkers in the diagnosis of disease cases, or in following disease progression and response to treatment as well. Therefore the unavailability of reliable biomarkers is a serious shortcoming.

Given the multifactorial nature of the disease, it is unlikely that a single biomarker will meet the needs for clinical diagnosis; beside that it is a formidable task to reach the appropriate sensibility and sensitivity.

$\mathrm{AD}$ is considered a multifactorial disease because the postmortem pathological diagnosis of an AD brain relies on unspecific histological findings as the presence of senile plaques neurofibrillary tangles. These senile plaques are composed of $\beta$-amyloid $(A \beta)$, a proteolytic fragment of the Amyloid precursor Protein (APP). An altered proteolytic processing of APP implies that several biochemical processes (known and unknown) must take place in a highly complex ordered form, perhaps way beyond our abstraction capacity.

Many molecular lesions have been detected in Alzheimer's disease, seems as accumulation of misfolded proteins in the aging brain results in oxidative and inflammatory damage, which in turn leads to energy failure and synaptic dysfunction. Brain cells are highly energy dependent for maintaining ion homeostasis during high metabolic activity [7]. Decreasing brain metabolism (from Greek metabolikós: changeable, metabole: change, metaballein, to change) is a significant cause of cognitive abnormalities of $\mathrm{AD}$.

Synapses are the first to show pathological variations in AD before the onset of clinical symptoms, because synaptic function has high energy demands. There is an age-related decline in neuronal NADH. Due to energy low levels, neuronal susceptibility to damage as a function of age and age related disease becomes important. In rat neuron model, mitochondria tend to be chronically depolarized, thereby producing more reactive oxygen species with age.

The already described pathogenic pathways leading to 
Citation: Herrera AS, Leszek J, del Carmen Arias Esparza M, Solís-Arias RI, Solís-Arias PE, et al. (2013) Human Photosynthesis: A Turning Point in the Understanding and Treatment of Alzheimer's Disease. J Bioanal Biomed 5: 057-060. doi:10.4172/1948-593X.1000079

neurodegeneration in $\mathrm{AD}$ include accumulation of aberrant or misfolded proteins, ubiquitin-proteasome system dysfunction, excitotoxic reactions, oxidative and nitrosative stress, mitochondrial injury synaptic failure, altered metal homeostasis, dysfunction of axonal and dendritic transport [8].

The major obstacle in managing the disease and designing rational therapeutic targets is our incomplete understanding of the pathogenesis of the disease. Several hypotheses have been proposed in an attempt to explain $\mathrm{AD}$ pathogenesis, including: amyloid deposition, tau phosphorylation, oxidative stress, metal ion dysregulation, and inflammation. No single one of these theories is sufficient to explain the spectrum of abnormalities found in the disease. However, the long-awaited answer about the fundamental initiator of the pathophysiological cascade in $\mathrm{AD}$ come to an end with the unraveling of the intrinsic property of melanin to split and re-form the water molecule.

If the available intracellular free chemical energy of the neuronal tissue is not sufficient for their incessant metabolic needs, sooner or later the affected organ or system become disorganized by a generalized failure being the hallmark of multifactorial disease. Brain energy metabolism is mainly centered in neuronal energy metabolism; however, other brain cells such as glial and vascular endothelial cells play an active role in the flux of biomass substrates to neurons. Recall that the basal rate of glucose utilization in astrocytes is higher than in neurons [9-11]. Glucose is the primary substrate for the buildup of biomass in brain tissue. By other side, energy is taken from the water of CSF.

Mitochondria are positioned within axons, dendrites, and synaptic terminals [12] to keep low phosphates levels through ATP synthesis and also serve as calcium buffering for these compartments. Synaptic compartments are regions of neurons exposed to highest levels of oxidative and metabolic stress, thereby in presence of low levels of the best antioxidant: the molecular hydrogen, synapses are the sites where the neurodegenerative process occurs early in $\mathrm{AD}$.

\section{Water as Source of Energy}

The dissociation of the water molecule can be schematized as follows:

$$
2 \mathrm{H}_{2} \mathrm{O} \leftrightarrow 2 \mathrm{H}_{2}+\mathrm{O}_{2}+4 \mathrm{e}^{-}
$$

This reaction was termed Solis-Herrera cycle by its discoverer. When the water molecule is broken, then oxygen and hydrogen diatomic is released; but the most valuable reaction product is diatomic or molecular hydrogen due to be the energy carrier by excellence in the entire universe. By other hand, molecular oxygen, one of the most stable molecules already known, is toxic at any level. The dissociation of the water molecule is very expensive from energetic point of view, since in the laboratory the same process requires heat up the water until $2000^{\circ} \mathrm{C}$.

Besides to be the energy carrier by excellence, diatomic hydrogen has other very valuable characteristic: it's the best antioxidant; for instance is able to reduce the oxygen itself, forming water producing at the same time 4 high energy electrons for each two water molecules re-formed.

\section{The Water of the CSF is the Main Source of Energy of the Central Nervous System}

Melanin has the intrinsic property to dissociate and re-form the water molecule, and was termed as human photosynthesis by analogy with the intrinsic property of chlorophyll to split irreversible the water molecule. Choroid plexus means grape color due to its elevated melanin content. Thereby, melanin has a fundamental role in the CSF production being regulated in turn by the available photonic (from light) energy.

The anatomical characteristics of the cerebral cortex are very consistent with our theory that the water of the CSF is the real source of energy of CNS, by other side blood vessels are the provider of the building blocks of the CNS biomass.

The cerebral sulcus is the way in which Nature ensure a close and continuous contact between the source of energy (water of CSF) and neuronal tissue (Figure 2). Thereby the real source of energy of the CNS are the ventricles and subarachnoid space. Blood vessels are just source of biomass beside the drive of $\mathrm{CO}_{2}$, a dangerous compound whose elevated levels out of normal range in tissues and liquids of the body are able to weaken marked and rapidly the turnover rate of dissociation and re-formation of the water molecule, causing death in less of one minute.

The turnover rate in ewes changes according to the light-dark cycle; it is increased during short day periods and reduced in long-day period. Recall that the first step of the human photosynthesis, the water dissociation, is the most expensive part of the Solis-Herrera cycle from the energetic point of view. Thereby it is favored by increase in amount of light. In other words: more energy = less liquid water in average (more dissociation).

\section{Comment}

Degeneration of synapses correlates strongly with cognitive decline [13-16]. Synaptic loss is associated through reduction in metabolic activity, which can be expected from low level of photosynthesis; abnormal activation of microglia and neuronal-astrocytic interactions are also impaired during $\mathrm{AD}$. The basic question about where does synaptic pathology start can be answered with human photosynthesis. Characteristically low levels of energy has a diffuse negative effect in cell metabolism, thereby numerous intracellular processes are impaired in a random-like manner.

Distal dendrites are first to be affected before the appearance of neurofibrillary tangles [2]. Synaptic plasticity is highly dependent on the capacity of neurons to meet energy demands to maintain hydric balance throughout ionic homeostasis, taking into account that approximately 100 times the volume of water in a cell crosses the

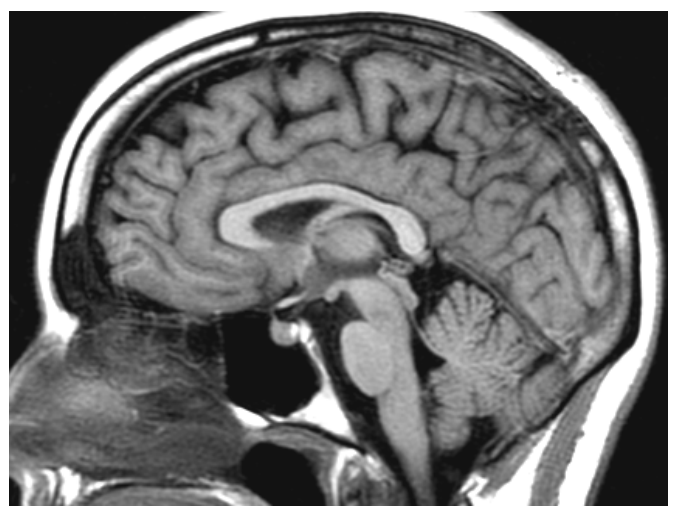

Figure 2: The cerebral sulcus. 
Citation: Herrera AS, Leszek J, del Carmen Arias Esparza M, Solís-Arias RI, Solís-Arias PE, et al. (2013) Human Photosynthesis: A Turning Point in the Understanding and Treatment of Alzheimer's Disease. J Bioanal Biomed 5: 057-060. doi:10.4172/1948-593X.1000079

plasma membrane every second [3]. Events associated with energy balance can impact synaptic and cognitive function [2]. An effective synaptic-preserving therapy must comprise numerous therapeutic targets given the complexity of the involved processes; nevertheless, energy enhancement measures have been neglected so far due to collective mistake about glucose. However evidence is overwhelming: glucose is just the source of biomass and water is the real source of energy, a conceptual revolution of biblical proportions.

Melanin characteristically tends to surround the cell nucleus, and is strategically placed in the perinuclear space as shown in Figure 3.

The optic nerve, a minute structure (1200 microns); notice in this photograph (Figure 4) the everpresence of melanin (dark rim) in 3-12 meridians.

The energy of the daylight determines dopamine.

\section{Biography}

Dr. Solis-Herrera graduated as MD from National Polytechnic Institute from Mexico, D.F.; and had the Ophthalmologist title from UNAM, (Universidad NacionalAutónoma de México); NeuroOphthalmologist from National Institute of Neurology, Master in Medical Sciences from University of Aguascalientes and $\mathrm{PhD}$ in Pharmacology from University of Guadalajara. Dr. Arturo Solis Herrera is director and founder (October 7, 2008) of Human Photosynthesis Study Center, in Aguascalientes, México. Dr. Solis-Herrera discovered the intrinsic property of melanin to split and reform the water molecule during a study about the three main causes of blindness in the world initiated in 1990. Finally, in February 2002; Dr. Solís-Herrera could understand that melanin's real function in human body is the

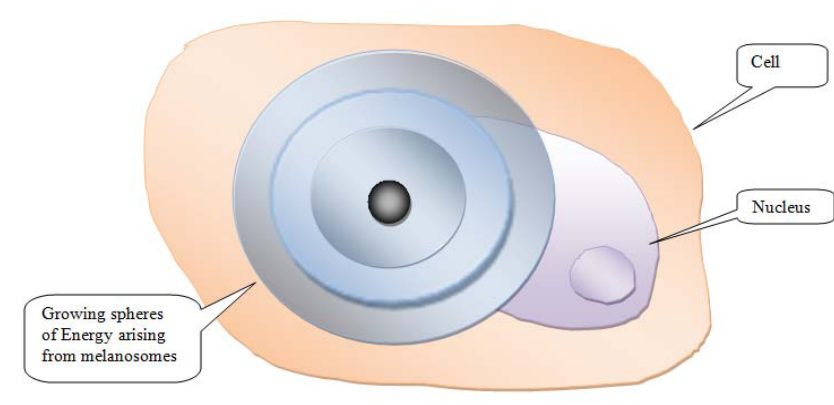

Figure 3: Melanin characteristically tend to surround the cell nucleus, and is strategically placed in the perinuclear space.

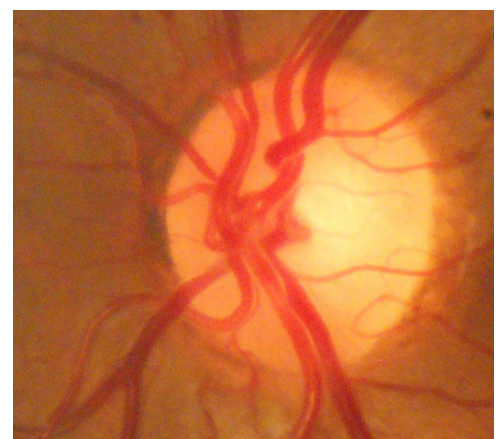

Figure 4: The optic nerve, a minute structure. transformation of the visible and invisible light (photonic energy) into chemical energy throughout the dissociation of the water molecule; a very expensive chemical reaction from the energetic point of view. This is nothing less that equivalent in human beings to the photosynthesis in plants; a comparable discovery to the understanding of the earth translation movements and of the roundness of our planet.

\section{References}

1. Nickelsen K (2007) Otto Warburg's first approach to photosynthesis. Photosynth Res 92: 109-120.

2. Craig $P$ (2005) Centennial history of the Carnegie Institution of Washington, vo IV: The Department of Plant Biology. Cambridge University Press, Cambridge.

3. Davide D, Pouya J, Stefa L (2013) Spitzer Nicholas C. Neurotransmitter Switching in the Adult Brain Regulates Behavior. Science 340: 449-453.

4. Solis Herrera A, Arias Esparza MC, Solis Arias RI, Solis Arias P, Solis Arias MP (2010) The Unexpected Capacity of Melanin to Dissociate the Water Molecule fills the Gap between the Life Before and After ATP. Biomedical Research 21 221-224

5. Sidell BD (1998) Intracellular Oxygen Diffusion: The Roles of Myoglobin and Lipid at Cold Body Temperature. The J Exp Biol 201: 1118-1127.

6. Thiéry JC, Lomet D, Sylvain B, Malpaux B (2009) Turnover rate of cerebrospinal fluid in female sheep: changes related to different light-dark cycles. Cerebrospinal Fluid Res 6: 9.

7. Henry WQ, La Frank ML (2010) Alzheimer's Disease. N Engl J Med 362: 329344.

8. Craig-Shapiro Rebecca, Fagan Anne M, Holztman David M (2009) Biomarkers of Alzheimer's Disease. Neurobiol Dis 35: 128-140.

9. Mordhwaj SP, Gregory J Brewer (2007) Mitoenergetic failure in Alzheimer's Disease. Am J Physiol Cell Physiol 292: C8-C23.

10. Cacabelos R, Fernandez NL, Lombardi V, Kubota Y, Takeda M (2005) Molecular Genetics of Alzheimer's Disease and Aging. Methods Find Exp Clin Pharmacol Suppl A: 1-573.

11. Magistretti PJ, Pellerin L (1996) Cellular bases of brain Energy metabolism and their relevance to functional brain imaging: evidence for a prominent role of astrocytes. Cereb Cortex 6: 50-61.

12. Mattson MP, Liu D (2003) Mitochondrial potassium channels and uncoupling proteins in synaptic plasticity and neuronal cell death. Biochem Biophys Res Commun 304: 539-549.

13. DeKiaky ST, Scheff SW, Styren SD (1996) Structural correlates of cognition in dementia: quantification and assessment of synapse change. Neurodegeneration 5: 417-421.

14. Braak H, Braak E, Strothjohann M (1994) Abnormally phosphorylated tau protein related to the formation of neurofibrillary tangles and neuropil threads in the cerebral cortex of sheep and goat. Neurosci Lett 171: 1-4.

15. Sherwood Lauralee. Human Physiology, from cells to systems. Thomson / Brooks/Cole Ed (5thedn). Australia 78.

16. Liu J, Head E, Gharib AM, Yuan W, Ingersoll RT, et al. (2002) Memory loss in old rats is associated with brain mitochondrial decay and RNA/DNA oxidation: partial reversal by feeding acetyl-L-carnitine and/or R-a-lipoic acid. Proc Nat Acad Sci 99: 2356-2361. 